\title{
Hydrogen Sulfide Inhibits Human T-cell Leukemia Virus Type-1 (HTLV-1) Protein Expression via Regulation of ATG4B
}

Liu Huandi ( $\square$ huandi1984@163.com )

Xinxiang Medical University https://orcid.org/0000-0003-4242-2335

Jiaxiang Sun

Xinxiang Medical College: Xinxiang Medical University

Shuaifeng Guo

Xinxiang Medical University

Xuhong Cheng

Shanghai Seventh People's Hospital

Zhongxin Zhang

Xinxiang Medical University

Jia Wan

Xinxiang Medical University

Chunduo Wang

Xinxiang Medical University

Xiaoying Zhi

Xinxiang Medical University

Linghui Yuan

Xinxiang Medical University

Hui Wang

Xinxiang Medical University

\section{Research Article}

Keywords: human T-cell leukemia virus type 1, hydrogen sulfide, ATG4B autophagy

Posted Date: December 22nd, 2021

DOI: https://doi.org/10.21203/rs.3.rs-1169449/v1

License: (1) (1) This work is licensed under a Creative Commons Attribution 4.0 International License.

Read Full License 


\title{
Hydrogen sulfide inhibits human T-cell leukemia virus type-1 (HTLV-1) protein expression via regulation of ATG4B
}

Huandi Liu ${ }^{1,2}$, Jiaxiang Sun ${ }^{1,2}$, Shuaifeng Guo ${ }^{2}$, Xuhong Cheng ${ }^{3}$, Zhongxin Zhang ${ }^{2}$, Jia Wan $^{2}$, Chunduo Wang ${ }^{2}$, Xiaoying Zhi' ${ }^{2}$, Linghui Yuan ${ }^{2}$, Hui Wang ${ }^{1,2}$

\author{
${ }^{1}$ Henan Key Laboratory of Immunology and Targeted Drugs, School of Laboratory Medicine, Xinxiang Medical \\ University, Xinxiang, China. \\ ${ }^{2}$ Henan Collaborative Innovation Center of Molecular Diagnosis and Laboratory Medicine, Xinxiang Medical \\ University, Xinxiang, China. \\ ${ }^{3}$ Seventh People's Hospital of Shanghai University of Traditional Chinese Medicine, Shanghai, China. \\ Correspondence and requests for materials should be addressed to H.W. (email: wanghui@xxmu.edu.cn)
}

\begin{abstract}
:
Background: Hydrogen sulfide $\left(\mathrm{H}_{2} \mathrm{~S}\right)$ is a redox gasotransmitter. It has been shown that $\mathrm{H}_{2} \mathrm{~S}$ has a key role in host antiviral defense by inhibiting interleukin (IL)-6 production and S-sulfhydrating Keap1 lead to Nrf2/ARE pathway activation. However, it is yet unclear whether $\mathrm{H}_{2} \mathrm{~S}$ can play an antiviral role by regulating autophagy.

Results: In this research, we found that exogenous $\mathrm{H}_{2} \mathrm{~S}$ decreased the expression of HTLV-1 protein and HTLV-1 induced autophagosomes accumulation. Transmission electron microscope assays indicated that autophagosomes accumulation decreased after $\mathrm{H}_{2} \mathrm{~S}$ administration. HTLV-1-transformed T-cell lines had a high level of CSE $\left(\mathrm{H}_{2} \mathrm{~S}\right.$ endogenous enzyme) which could be induced in Hela by HTLV-1 infection. Immunoblot demonstrated that overexpression of CSE inhibited HTLV-1 protein expression and autophagy. And we got the opposite after CSE knockdown. Meanwhile, $\mathrm{H}_{2} \mathrm{~S}$ could not restrain the autophagy when ATG4B had a mutant at its site of 89 .

Conclusion: In a word, these results suggested that $\mathrm{H}_{2} \mathrm{~S}$ modulated HTLV-1 protein expression via ATG4B. Meanwhile, our findings suggested a new mechanism by which $\mathrm{H}_{2} \mathrm{~S}$ defended against virus infection.
\end{abstract}

Key words: human T-cell leukemia virus type 1 hydrogen sulfide ATG4B autophagy

\section{Background:}

$\mathrm{H}_{2} \mathrm{~S}$, hydrogen sulfide, is a colorless gas with lipid soluble. It has a smell of rotten eggs and has been supposed to be a kind of poisonous gas previously. Lately, it has been reported that $\mathrm{H}_{2} \mathrm{~S}$ could be produced in mammalian cells ${ }^{1-4}$. There are different concentration of $\mathrm{H}_{2} \mathrm{~S}$ in different part. For example, it is about $46 \mu \mathrm{mol} \cdot \mathrm{L}^{-1}$ in rat serum and $100 \mu \mathrm{mol} \cdot \mathrm{L}^{-1}$ in rat brain ${ }^{5-6}$. Endogenous $\mathrm{H}_{2} \mathrm{~S}$ is produced in the cells by three enzymes, CSE (cystathionine $\gamma$-lyase), CBS (cystathionine $\beta$-synthase) and MST (3mercaptopyruvate sulfur transferase $)^{7-9}$. CBS is the main enzyme in the central nervous system $^{6,10,11}$, CSE is the key enzyme in the cardiovascular system ${ }^{12,13}$, whereas MST is responsible for the production of $\mathrm{H}_{2} \mathrm{~S}$ in the kidney ${ }^{14,15}$. In addition, $\mathrm{H}_{2} \mathrm{~S}$ has a pivotal role in digestive system and respiratory system ${ }^{16-19}$. Consequently, $\mathrm{H}_{2} \mathrm{~S}$ is considered as the third gasotransmitter, such as carbon monoxide (CO) and nitric oxide (NO) ${ }^{20-22}$. 
Sulfhydration is the main mechanism of $\mathrm{H}_{2} \mathrm{~S}$ in regulating protein activity ${ }^{23,24}$. In this modification, $\mathrm{H}_{2} \mathrm{~S}$ changes a Cys residue $(-\mathrm{SH})$ of a target protein into $-\mathrm{SSH}^{23}$, ${ }^{24}$. For example, $\mathrm{H}_{2} \mathrm{~S}$ can restain Keap1 activity by sulfhydration of Cys 151 , and then Nrf2 translocates into the nucleus and combine with Antioxidant Response Element (ARE) ${ }^{25,26}$. This results in the expression of antioxidant genes.

Autophagy is a degradation process in cellular that transmits cytoplasmic components to lysosomes via autophagosomes ${ }^{27,28}$. Lipidation of LC3 is important for early stage of autophagy membrane formation ${ }^{29,30}$. ATG4, a kind of cysteine protease, perform as executer to process LC3 lipidation and delipidation of lipidated LC $3^{31,32}$. There are four ATG4 isoforms in mammal cells, such as ATG4A, ATG4B, ATG4C and ATG4D ${ }^{33}$. ATG4B is the key isoform for cleaving LC3/GABARAP ${ }^{34}$. ATG4A has the ability of processing GABARAP. Compared with ATG4B, its activity is weak ${ }^{35}$. However, ATG4D and ATG4C have almost no activity in vitro ${ }^{34,36}$.

Adult T-cell leukemia (ATL), a hematologic malignant disease of adult, is directly related to the infection of human T-cell leukemia virus type 1 (HTLV-1) $)^{37-39}$. It has a variety of symptoms like acute type, chronic type or insidious type ${ }^{40,41}$. Chronic and insidious patients are often supported by symptomatic support treatment; Chemical and biological therapy are often used to cure acute lymphoma. However, the effect is unsatisfactory, and the median survival of the acute patients is about $2 \sim 6$ months ${ }^{40,42-}$ 44 .

Tax, HTLV-1 protein, can continuously activate autophagy and generate a lot of autophagosomes. And then, HTLV-1 is wrapped in the autophagosomes. HTLV-1 in autophagosomes not only can get away from intracellular immunity but also complete self-replication in autophagosomes ${ }^{45,46}$. The massive virus promoted the expression of Tax. Lots of Tax continue to activate autophagy and advance virus replication via positive feedback, resulting in the generation and development of ATL finally ${ }^{47}$.

In this research, we demonstrated that CSE was induced by HTLV-1 infection and suppressed HTLV-1 protein expression. We showed that exogenous $\mathrm{H}_{2} \mathrm{~S}$ inhibited the accumulation of autophagosomes during HTLV-1 infection. Furthermore, we found that CSE and exogenous $\mathrm{H}_{2} \mathrm{~S}$ were associated with ATG4B which is essential for autophagy early period. Collectively, our findings may shed some new lights on HTLV-1 therapy and contribute to our understanding of $\mathrm{H}_{2} \mathrm{~S}$ defenses against virus infection.

\section{Results}

\section{$\mathrm{H}_{2} \mathrm{~S}$ treatment suppressed HTLV1 replication, reduces autophagy level, and has no effect on cells' viability.}

The increase of autophagy is a common manifestation of viral infection. HTLV-1 induces autophagy and complete their replication in autophagosomes. $\mathrm{H}_{2} \mathrm{~S}$, an endogenous gas signal molecule, can inhibit autophagy. That's the reason we explore the relationship between HTLV-1 infection and $\mathrm{H}_{2} \mathrm{~S}$. HTLV-1 positive cells, MT2, MT4 and C8166, have high level of autophagy. LC3 has two isoforms, LC3A (upper line) and LC3B (below line). LC3B is a marker of autophagy ${ }^{48}$. The expression of LC3B in MT2 reduced after $\mathrm{H}_{2} \mathrm{~S}$ administration for 48 hours (Fig. 1A.), and virus protein Tax and p19 also decreased. We saw the same results in MT4 (Fig. 1B.) and C8166 (Fig. 1C.). Meanwhile, the level of Tax gene expression was inhibited by $\mathrm{H}_{2} \mathrm{~S}$ (Fig. 1D.) 
significantly. In order to further determine whether the decrease of viral protein expression is related to the decrease of cell activity, MTT assay was performed. As shown in Fig. 1E, $\mathrm{H}_{2} \mathrm{~S}$ had no effect on MT2 viability for 48 hours. And the cells viability increased after $\mathrm{H}_{2} \mathrm{~S}$ administration for 72 hours. Consistently, the accumulation of autophagosomes was decreased in $\mathrm{H}_{2} \mathrm{~S}$-treated MT2 cells compared to MT2 without $\mathrm{H}_{2} \mathrm{~S}$ administration (Fig. 1F). These results suggests that the effect of $\mathrm{H}_{2} \mathrm{~S}$ on HTLV-1 protein expression was associated with autophagy.
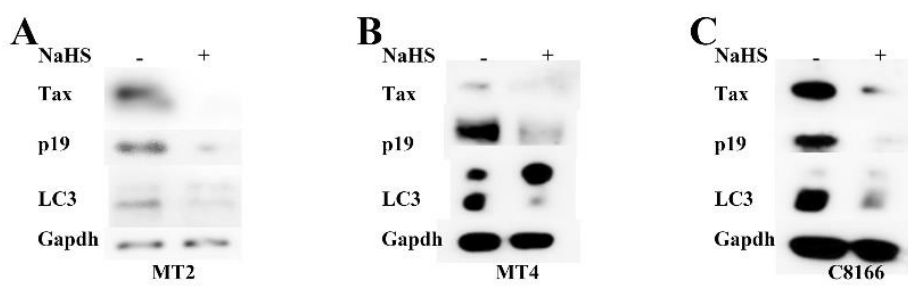

D
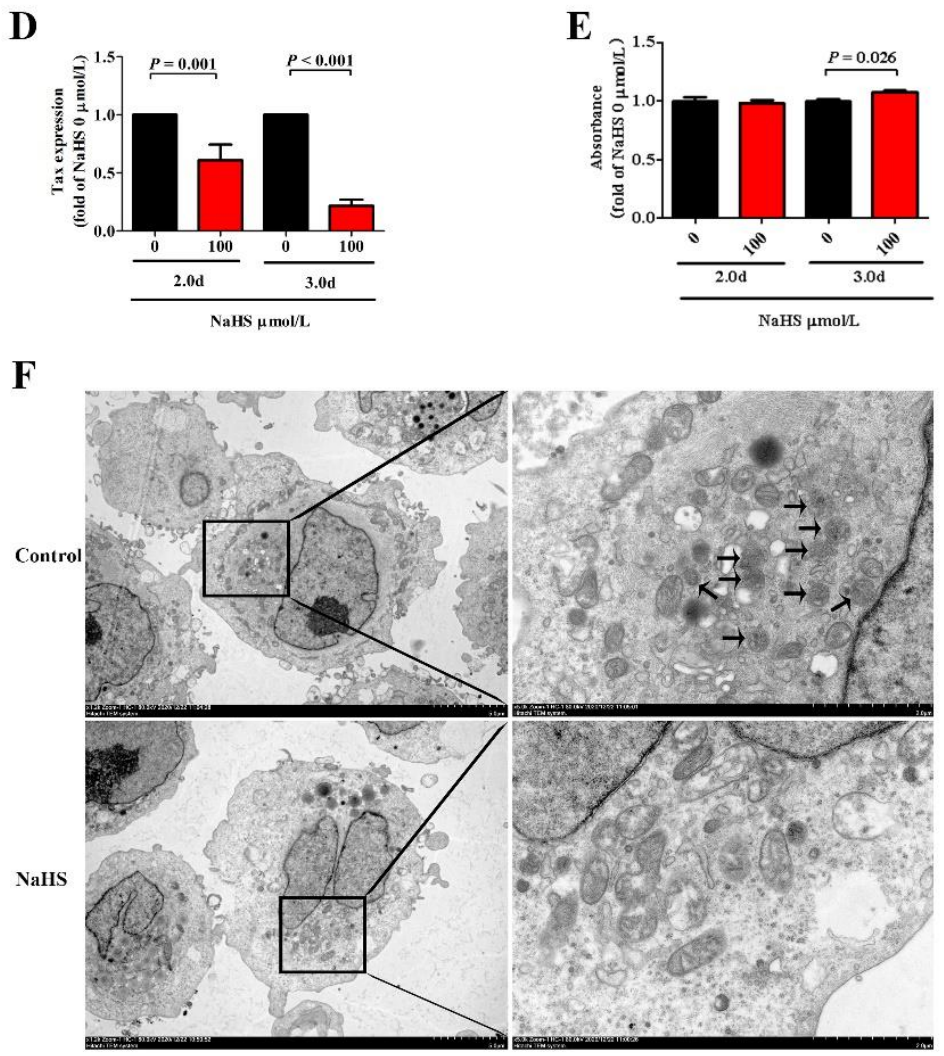

Figure 1 The inhibition of virus replication by H2S was related to autophagy. MT2 cells (A), MT4 (B) and C8166 cells (C) were stimulated by NaHS. After $48 \mathrm{~h}$, the cell lysates were immunized with anti-Tax, anti-p19, anti-LC3 as indicated. (D) MT2 cells were stimulated by NaHS. After $2 \mathrm{~d}$ or $3 \mathrm{~d}$, the cells were lysated for real-time PCR assay. (E) MT2 cells were stimulated by NaHS. After 2 days (d) or 3 days (d), MTT Cell Proliferation and Cytotoxicity Assay Kit was used as manufacturer's instructions. (F) MT2 cells were stimulated by NaHS. After $2 \mathrm{~d}$, the cells were fixed with the TEM fixative. Autophagosomes were indicated by the black arrows.

\section{Autophagy plays a key role in the inhibition of virus replication by $\mathrm{H}_{2} \mathrm{~S}$.}

We determined the role of $\mathrm{H}_{2} \mathrm{~S}$ in MT2 cells in the presence of RA (rapamycin) or 3- 
MA (3-Methyladenine), which are believed to activate or block the autophagy. We found that RA could reduce the effect of $\mathrm{H}_{2} \mathrm{~S}$, and 3-MA could enhance the effect of $\mathrm{H}_{2} \mathrm{~S}$ (Fig.2A). Similar results were observed in MT4 and C8166 (Fig. 2B and C). Meanwhile, NaHS could also restrain the autophagy of Hela (Fig. 2D). It means that $\mathrm{H}_{2} \mathrm{~S}$ depressed autophagy not only for HTLV-1 positive cells. And its effect on Hela laid a foundation for our further study of the mechanism.
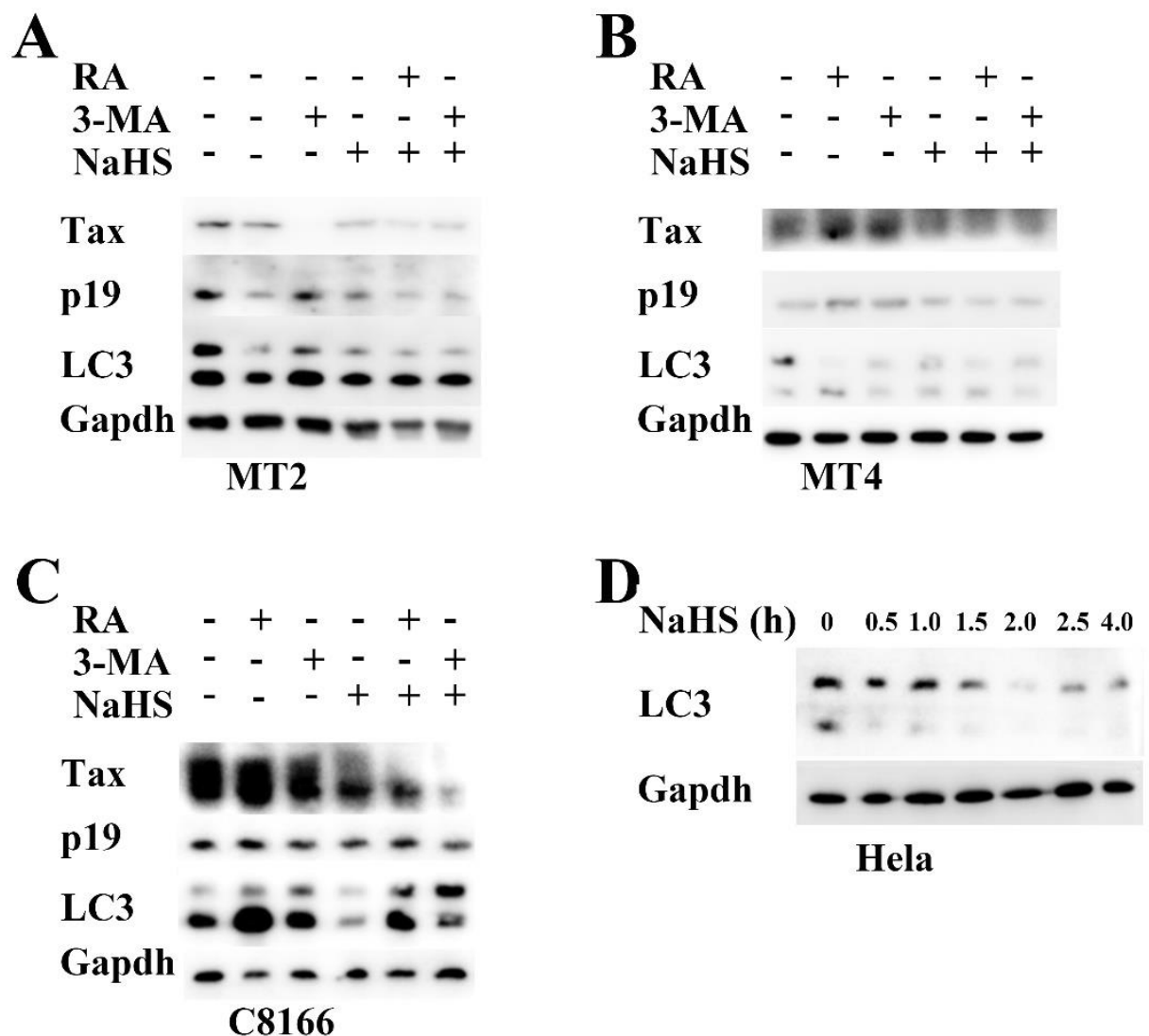

Figure 2 The effect of ATG4B in $\mathrm{H}_{2} \mathrm{~S}$-induced suppression of HTLV1 replication. MT2 cells (A), MT4 (B) and C8166 cells (C) were stimulated by NaHS, RA, 3-MA, or both two. After 48 h, the cell lysates were immunized with anti-Tax, anti-p19, anti-LC3 as indicated. (D) Hela cells were stimulated by NaHS for several different time. The cell lysates were immunized with anti-LC3.

\section{$\mathrm{H}_{2} \mathrm{~S}$ interacts with ATG4B.}

To characterize the mechanism by which $\mathrm{H}_{2} \mathrm{~S}$ regulated autophagosome accumulation, we tested whether $\mathrm{H}_{2} \mathrm{~S}$ was able to interact with ATG4B involved in canonical autophagy. ATG4B is a cysteine proteinase, which activity dependents on the cysteine located in the active center ${ }^{49}$. ATG4B has twelve cysteines according to NCBI database (Fig. 3). It has been reported that ATG4B has no activity if the cysteine at site 74 is mutated $^{50,51}$. Meanwhile, $\mathrm{H}_{2} \mathrm{~S}$ signals is by modification of cysteine residues on target proteins in a process designated as sulfhydration or persulfidation. Therefore, we constructed 11 mutants which had one cysteine mutated separately except the site 74 . The plasmid of ATG4B and its cycsteine mutants were transfected in Hela cells. NaHS was administrated. To confirm the effect of these mutants on ATG4B activity, Hela cells were treated by RA or 3-MA and then subjected to immunoblot analysis. The results 
showed that RA and 3-MA could active or depress autophagy when ATG4B had these cysteine mutated (Fig. 4A and 4B). It suggested that these cysteine were not essential for ATG4B activity. However, $\mathrm{H}_{2} \mathrm{~S}$ had no effect on autophagy when the cysteine at site 89 was mutated (Fig. 4C). These data indicated that cysteine 89 of ATG4B was the target of $\mathrm{H}_{2} \mathrm{~S}$ in the regulation of autophagy.

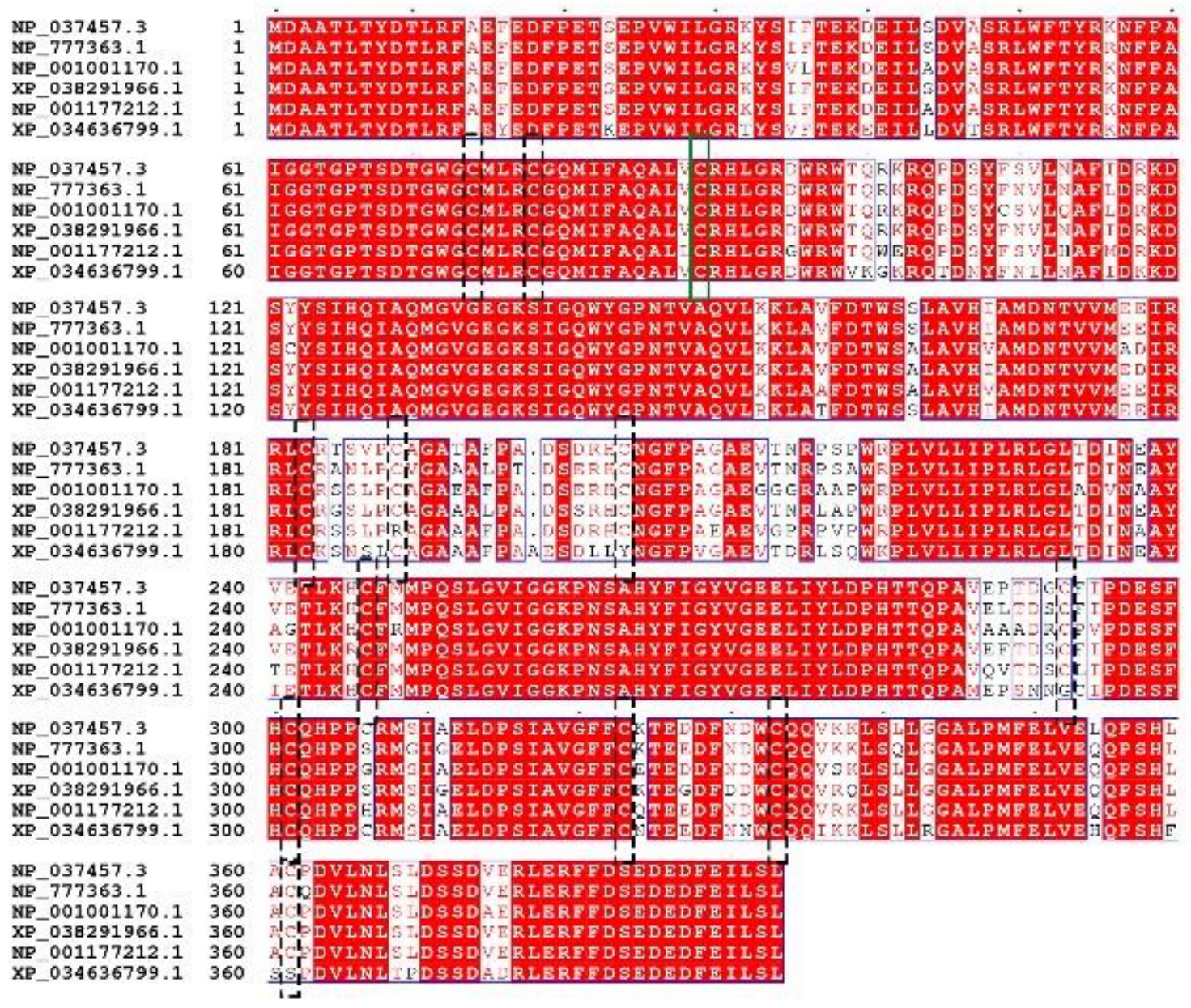

Figure 3 Alignment of the amino acid sequence of ATG4B and its homologues in human and other species. Sequence of human (NCBI Reference Sequence: NP_037457.3), mouse (NCBI Reference Sequence: NP_777363.1), bovine (NCBI Reference Sequence: NP_001001170.1), dog (NCBI Reference Sequence: XP_038291966.1), pig (NCBI Reference Sequence: NP_001177212.1), and trachemys scripta elegans (NCBI Reference Sequence: XP_034636799.1) were compared by ESPRIPT $3 . \mathrm{x}^{52}$. Twelve cysteine sites, marked by box, are highly conservative. 
A

RA

Ath4B (C/A)con Vek $78 \quad 89183189203246293302323333361$

Ath4B - -

LC3

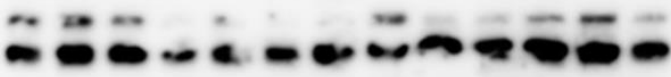

Gapdh

B

3-MA

Ath4B (C/A)con Vek $78 \quad 89183189203246293302323333361$

Ath4B

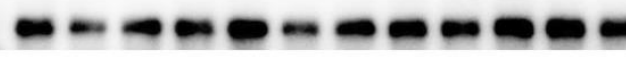

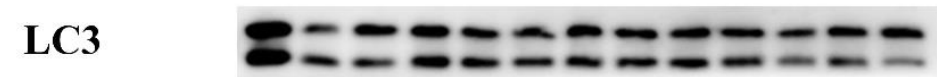

Gapdh

$--0-0-0-0$

C

NaHS - ++++++++++++

Ath4B (C/A)con Vek $78 \quad 89183189203246293302323333361$

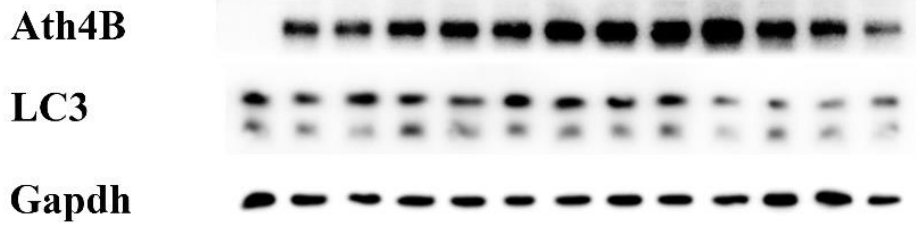

Figure 4 The effect of ATG4B in $\mathrm{H}_{2} \mathrm{~S}$-induced suppression of autophagy. (A) Hela cells were transfected with ATG4B or its cysteine mutants. After $48 \mathrm{~h}$, the cells were stimulated by RA. The cell lysates were immunized with anti-LC3 as indicated. (B) Hela cells were transfected with ATG4B or its cysteine mutants. After $48 \mathrm{~h}$, the cells were stimulated by 3-MA. The cell lysates were immunized with anti-LC3 as indicated. (C) Hela cells were transfected with ATG4B or its cysteine mutants. After $48 \mathrm{~h}$, the cells were stimulated by NaHS. The cell lysates were immunized with antiLC3 as indicated.

\section{CSE expression increases in early period of HTLV-1 infection.}

Then we tried to explore the functions of $\mathrm{H}_{2} \mathrm{~S}$ endogenous enzyme during HTLV-1 infection. Jurkat and MT2 cells were lysated, and $\mathrm{H}_{2} \mathrm{~S}$ endogenous enzymes were investigated. Immunoblot assays demonstrated that the expression of $\mathrm{H}_{2} \mathrm{~S}$ endogenous enzymes were associated with HTLV-1 infection (Fig. 5A). Hela cells were co-cultured with MT2 cells and the expression levels of Tax was investigated. PCR assays demonstrated that Tax could be detected in Hela cells after co-cultured for $24 \mathrm{~h}$ (Fig. 5B). The expression of CSE reached a maximum on the $48 \mathrm{~h}$ and the level of autophagy decreased after co-culture (Fig. 5C). However, the expression of CBS and MST 
decreased after co-culture for $48 \mathrm{~h}$ (Fig. 5C). These results suggested that CSE played a key role in early period of HTLV-1 infection. In order to confirm that the decreased of autophagy was associated with CSE and HTLV-1. Hela cells were cultured without FBS. Immunoblot assays found that the increase of CSE could not reduce the level of autophagy without HTLV-1 infection (Fig. 5D). And then, we detected the effect of exogenous $\mathrm{H}_{2} \mathrm{~S}$ on Hela cells. The result showed that exogenous $\mathrm{H}_{2} \mathrm{~S}$ had no effect on the expression of endogenous enzymes (Fig. 5E). In summary, the expression of CSE decreases the level of autophagy of HTLV-1 positive cells.
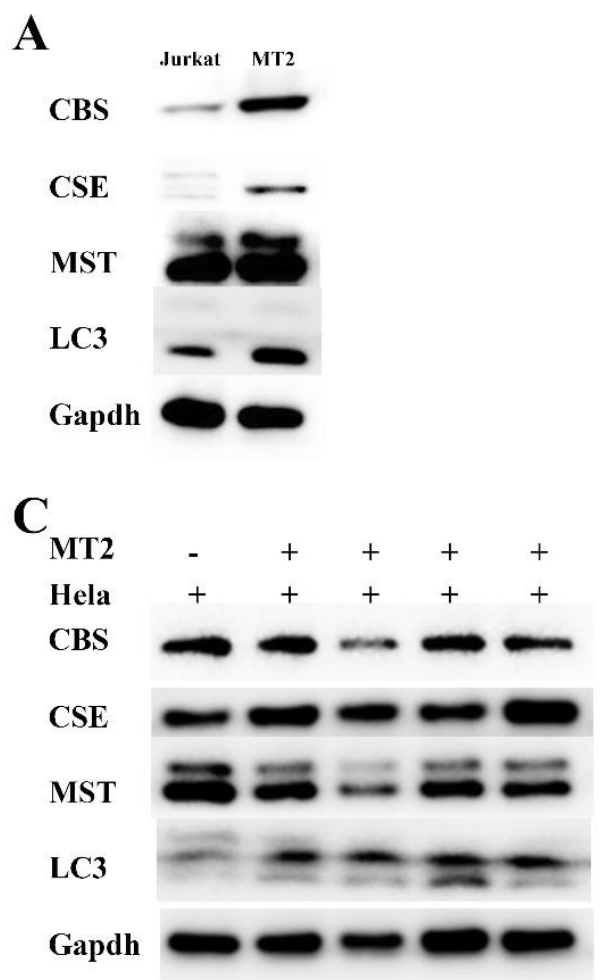

B

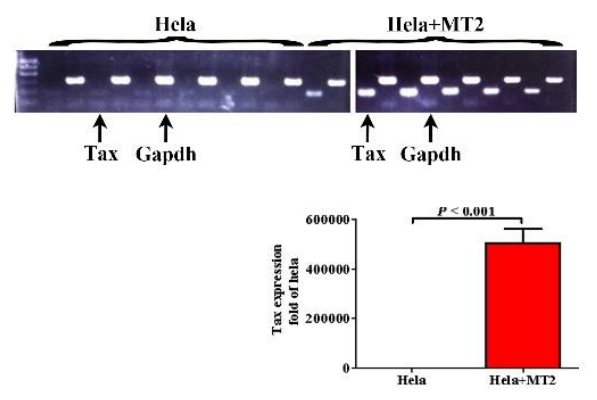

$\mathbf{D}$

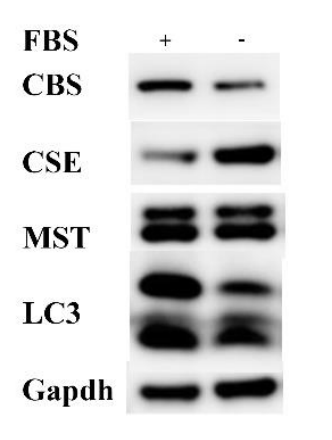

$\mathbf{E}$

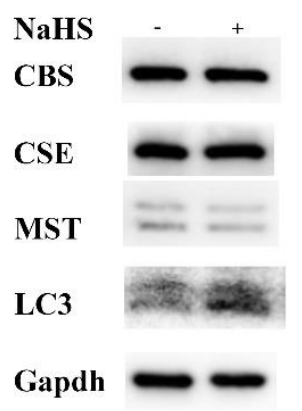

Figure 5 The effect of HTLV-1 infection on the expression of $\mathrm{H}_{2} \mathrm{~S}$ endogenous enzyme. (A) MT2 cells and Jurkat cells were lysated, and the lysates were immunized with anti-CBS, anti-CSE, antiMST, anti-LC3 as indicated. (B) Hela cells were seeded for $24 \mathrm{~h}$, and then co-cultured with MT2 cells for another $24 \mathrm{~h}$. The cells were lysated for PCR assay and agarose electrophoresis. (C) Hela cells were seeded for $24 \mathrm{~h}$, and then co-cultured with MT2 cells for another $24 \mathrm{~h}$. The cells lysates were immunized with anti-CBS, anti-CSE, anti-MST, anti-LC3 as indicated. (D) Hela cells were cultured without FBS for $48 \mathrm{~h}$. The cell lysates were immunized with anti-CBS, anti-CSE, anti-MST, anti-LC3 as indicated. (E) Hela cells were stimulated with NaHS. After 48 h, the cells lysates were immunized with anti-CBS, anti-CSE, anti-MST, anti-LC3 as indicated.

\section{CSE can inhibit HTLV-1 replication via ATG4B.}

Next, we wanted to make sure whether CSE had the same effect like exogenous $\mathrm{H}_{2} \mathrm{~S}$. Hela cells with overexpressing or low expressing of CSE were co-cultured with MT2 cells and the expression levels of HTLV-1 proteins and autophagy were investigated. Immunoblot assays demonstrated that the expression of CSE was associated with lower levels of HTLV-1 proteins and autophagy (Fig. 6A and 6B). Meanwhile, we investigated 
whether RA and 3-MA could impact the effect of CSE. We found that RA could reduce the effect of CSE, and 3-MA could enhanced the effect of CSE (Fig. 6C and 6D). In order to confirm that ATG4B was associated with the effect of CSE. Hela cells, overexpressing of CSE and ATG4B mutant, were co-cultured with MT2 cells. Immunoblot assays showed that the level of HTLV-1 protein was restored partly (Fig. $6 \mathrm{E})$. Taken together, these data suggested that the expression of CSE decreased HTLV1 protein via the cysteine 89 of ATG4B.
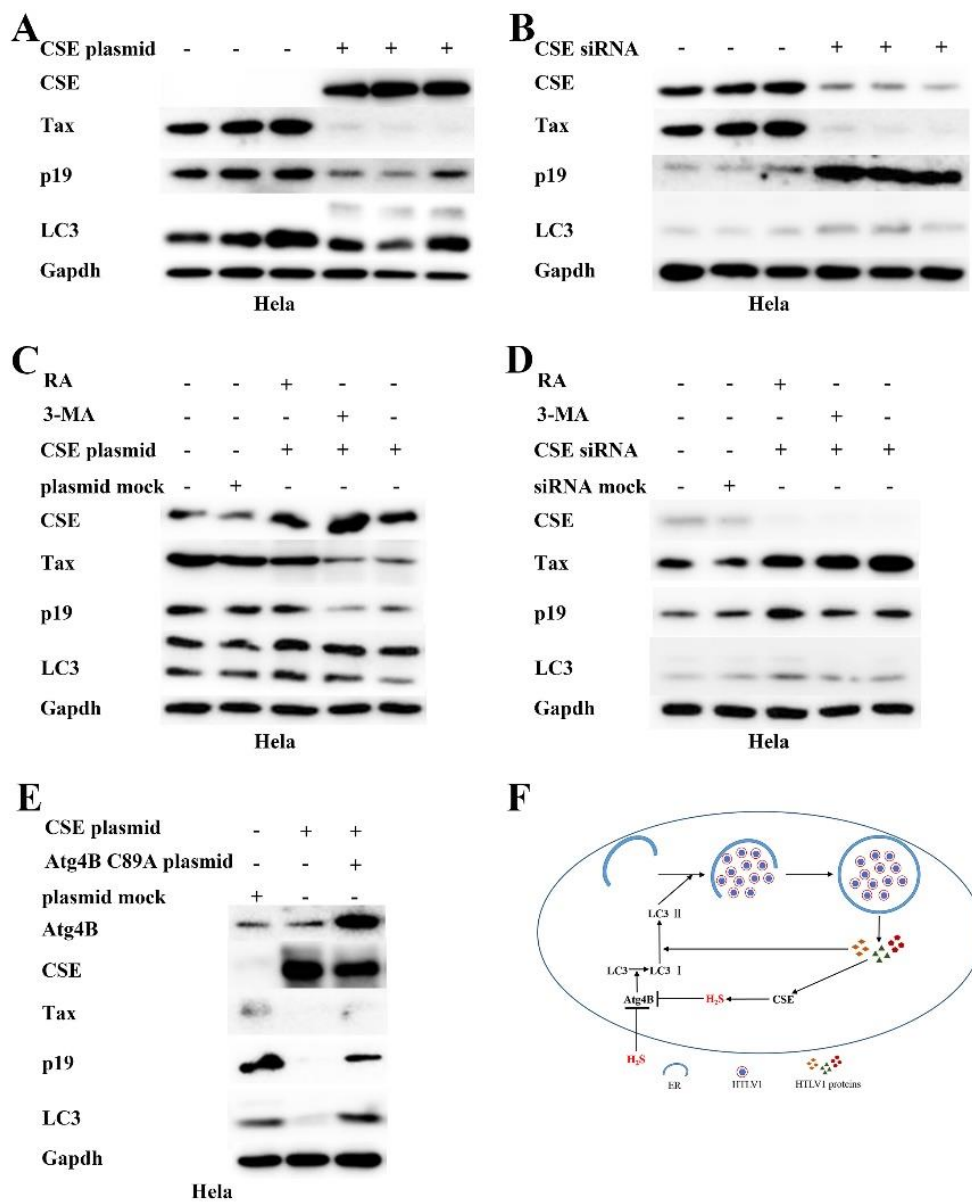

$\mathbf{F}$

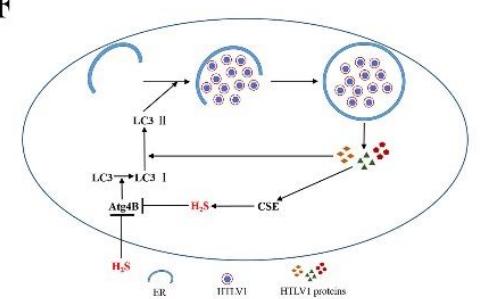

Figure 6 CSE can effectively regulate HTLV1 replication in early infection stage via ATG4B. (A) Hela cells were transfected with empty vector (-) or pCMV3-CSE (+). At $24 \mathrm{~h}$ after transfection, the cells were co-cultured with MT2 cells for another $24 \mathrm{~h}$. Then the cells were washed with PBS three times to remove MT2 cells and lysed for immunoblot assays. (B) Hela cells were transfected with negative control or CSE-siRNA. At $24 \mathrm{~h}$ after transfection, the cells were co-cultured with MT2 cells for another $24 \mathrm{~h}$. Then the cells were washed with PBS three times to remove MT2 cells and lysed for immunoblot assays. (C) Hela cells were transfected with empty vector (-) or pCMV3CSE (+). At $24 \mathrm{~h}$ after transfection, the cells were co-cultured with MT2 cells for another $24 \mathrm{~h}$. Then the cells were stimulated by RA or 3-MA for $24 \mathrm{~h}$. Finally, the cells were washed with PBS three times to remove MT2 cells and lysed for immunoblot assays. (D) Hela cells were transfected with negative control or CSE-siRNA. At $24 \mathrm{~h}$ after transfection, the cells were co-cultured with MT2 cells for another $24 \mathrm{~h}$. Then the cells were stimulated by RA or 3-MA for $24 \mathrm{~h}$. Finally, the cells were washed with PBS three times to remove MT2 cells and lysed for immunoblot assays. (E) Hela 
cells were transfected with empty vector (-) and negative control or pCMV3-CSE (+) and ATG4B C89A mutant. At $24 \mathrm{~h}$ after transfection, the cells were co-cultured with MT2 cells for another 24 $\mathrm{h}$. Then the cells were washed with PBS three times to remove MT2 cells and lysed for immunoblot assays. (F) The mechanism diagram of the effect among HTLV-1, autophagy, ATG4B, CSE and $\mathrm{H}_{2} \mathrm{~S}$. (1)HTLV-1 induced the formation of autophagosomes and enhanced HTLV-1 protein expression. (2) HTLV-1 increased the expression of CSE. (3) CSE expression and exogenous $\mathrm{H}_{2} \mathrm{~S}$ decreased autophagy accumulation and lower HTLV-1 protein expression levels via ATG4B.

\section{Discussion:}

As a gasotransmitter, $\mathrm{H}_{2} \mathrm{~S}$ plays an important role in the physiological and pathological process of the organism ${ }^{16-19}$. Nowadays, the mechanism of $\mathrm{H}_{2} \mathrm{~S}$ in the process of virus infection is unclear. It has been reported that $\mathrm{H}_{2} \mathrm{~S}$ could play an antiviral role through its antioxidant and anti-inflammatory effects, such as COVID-9, HSV, HIV and paramyxovirus ${ }^{53-58}$. Our study found that both endogenous and exogenous $\mathrm{H}_{2} \mathrm{~S}$ could inhibit autophagy caused by HTLV-1 and reduced the expression of viral proteins by autophagy related protein ATG4B.

Our results showed that HTLV-1-positive T-cell lines had a strong expression of CSE and CSE expression could be induced in Hela after HTLV-1 infection. Further studies indicated that the overexpression of CSE inhibited HTLV-1 protein expression and CSE knockdown was associated with higher levels of HTLV-1 protein expression. There was significant relevance between autophagy level and the expression of CSE. These results drove us to find other possible mechanism to explain the role of CSE in autophagy.

There is a violent war between autophagy and pathogenic microorganisms ${ }^{59}$. Paradoxically, autophagosomes is benefit for HTLV-1 to complete its replication ${ }^{45-47}$. We used LC3B as the marker of autophagy and examined the effect of CSE on HTLV1 induced autophagy. Our findings confirmed the hypothesis by three steps. Firstly, $\mathrm{H}_{2} \mathrm{~S}$ inhibited the autophagosomes accumulation in MT2 cells. Secondly, CSE inhibited the autophagosomes accumulation induced by HTLV-1 infection in Hela. Thirdly, when RA was used to activate the early stage of autophagy, we found that CSE overexpression lost the abilities to inhibit autophagosomes accumulation and HTLV-1 viral protein expression increased. These results suggested that the regulatory role of CSE in autophagosomes accumulation was important to its effect on HTLV-1 protein expression.

How could $\mathrm{H}_{2} \mathrm{~S}$ regulate autophagosomes accumulation? It has been reported that ATG4B has an important role in LC3 II and autophagosomes formation ${ }^{34}$. As a cysteine enzyme, ATG4B is a good target for $\mathrm{H}_{2} \mathrm{~S}$ regulation. Our findings indicated that $\mathrm{H}_{2} \mathrm{~S}$ interacted with ATG4B. Using a serious of ATG4B cysteine mutants, we found ATG4B cysteine 89 was essential for the effect of $\mathrm{H}_{2} \mathrm{~S}$. However, ATG4B will almost lost the ability when it has a mutant at the site of cysteine $74{ }^{60}$ 。 Therefore, we could not confirm whether the cysteine 74 was associated with $\mathrm{H}_{2} \mathrm{~S}$ function.

It is reported that the function of ATG4B can be changed via post-translational modification. For example, its activity is enhanced when the serine at 383 site was phosphorylated $^{61}$; After phosphorylation of serine at 34 and 316 sites or S-nitrosation 
of cysteine at 189 and 292 sites, the opposite results will appear ${ }^{62,63,64}$; In addition, when the level of reactive oxygen species increases, a disulfide bond will be formed between cysteines at site 292 and 361 resulting the level of autophagy increases ${ }^{65}$. Our study suggested that $\mathrm{H}_{2} \mathrm{~S}$ could sulfurize cysteine at site 89 and inhibit autophagy. However, we had no direct evidence to confirm the sulfhydration of ATG4B because of technical limitations.

Conclusion: Together, our study demonstrated that $\mathrm{H}_{2} \mathrm{~S}$ modulated autophagosomes accumulation by regulating ATG4B, thereby affecting the expression of HTLV-1 protein. Our research may expand our understanding of $\mathrm{H}_{2} \mathrm{~S}$ on virus infection and suggest a new role of ATGs sulfhydration in autophagy.

\section{Methods:}

\section{Regents}

The following antibodies were used for immunoblot analysis: anti-Gapdh (60004-1-Ig, Proteintech), anti-CBS (14782, Cell Signaling Technology), anti-CSE (19689, Cell Signaling Technology), antiHTLV-1 p19 (ab9080, Abcam), anti-MST (ab224043, Abcam), anti-Tax (sc-57872, Santa Cruz Biotechnology), anti-ATG4B (15131-1-AP, Proteintech), anti-LC3A/B (18725-1-AP, Proteintech). The 3-MA (M9281), NaHS (151627) and RA (553211) were obtained from Sigma-Aldrich. SYBR Green quantitative PCR master mix were purchased from TOYOBO Ideas and Chemistry (QPK201). MTT Cell Proliferation and Cytotoxicity Assay Kit was obtained from Beyotime Biotechnology.

\section{ATG4B mutants' preparation}

ATG4B plasmid was bought from Sino Biological (Cat. HG20407-ANG). GeneArt® Site-Directed Mutagenesis PLUS Kit was used to construct cysteine mutants. Gene-specific primer sequences were as follow:

C78A:

Forward: CACAGGCTGGGGCTGCATGCTGCGGGCTGGACAGATGATCTTTGCCCAAGCC

Reverse: GGCTTGGGCAAAGATCATCTGTCCAGCCCGCAGCATGCAGCCCCAGCCTGTG

C89A:

Forward: GATGATCTTTGCCCAAGCCCTGGTGGCCCGGCACCTAGGCCGAGATTGGAGG

Reverse: CCTCCAATCTCGGCCTAGGTGCCGGGCCACCAGGGCTTGGGCAAAGATCATC

C183A:

Forward: GTTGTGATGGAGGAAATCAGAAGGTTGCCAGGACCAGCGTTCCCTGTGCAGGC 
Reverse: GCCTGCACAGGGAACGCTGGTCCTGGCAACCTTCTGATTTCCTCCATCACAAC

C189A:

Forward: GAAGGTTGTGCAGGACCAGCGTTCCCGCTGCAGGCGCCACTGCGTTTCCTGCAG

Reverse: CTGCAGGAAACGCAGTGGCGCCTGCAGCGGGAACGCTGGTCCTGCACAACCTTC

C203A:

Forward: GTTTCCTGCAGATTCCGACCGGCACGCCAACGGATTCCCTGCCGGAGCTGAG

Reverse: CTCAGCTCCGGCAGGGAATCCGTTGGCGTGCCGGTCGGAATCTGCAGGAAAC

C246A:

Forward: GGCCTACGTGGAGACGCTGAAGCACGCCTTCATGATGCCCCAGTCCCTGGGC

Reverse: GCCCAGGGACTGGGGCATCATGAAGGCGTGCTTCAGCGTCTCCACGTAGGCC

C292A:

Forward: GCCAGCCGTGGAGCCCACTGATGGCGCCTTCATCCCGGACGAGAGCTTCCAC

Reverse: GTGGAAGCTCTCGTCCGGGATGAAGGCGCCATCAGTGGGCTCCACGGCTGGC

C301A:

Forward: CTTCATCCCGGACGAGAGCTTCCACGCCCAGCACCCGCCGTGCCGCATGAGC

Reverse: GCTCATGCGGCACGGCGGGTGCTGGGCGTGGAAGCTCTCGTCCGGGATGAAG

C323A:

Forward: CCCGTCCATCGCTGTGGGGTTTTTCGCTAAGACTGAAGATGACTTCAATGATTG

Reverse: CAATCATTGAAGTCATCTTCAGTCTTAGCGAAAAACCCCACAGCGATGGACGGG

C333A:

Forward: GACTGAAGATGACTTCAATGATTGGGCCCAGCAAGTCAAAAAGCTGTCTCTG

Reverse: CAGAGACAGCTTTTTGACTTGCTGGGCCCAATCATTGAAGTCATCTTCAGTC

C361A:

Forward: GGAGCTGCAGCCTTCACATCTGGCCGCCCCCGACGTCCTGAACCTGTCCCTAG

Reverse: CTAGGGACAGGTTCAGGACGTCGGGGGCGGCCAGATGTGAAGGCTGCAGCTCC

\section{Cell culture and transfection}

Hela cells was cultured in DMEM with high glucose. MT2, MT4, C8166 and Jurkat cells were grown in RPMI 1640. All cells were provided with 10\% FBS (Gibco), 4 mM L-glutamine under humidifed conditions with $5 \% \mathrm{CO}_{2}$ at $37{ }^{\circ} \mathrm{C}$. Transfection of Hela cells was performed with Lipofectamine 2000 (Invitrogen) according to the manufacturer's instructions. 


\section{Western blot}

Immunoblot analysis was performed. Hela cells were transfected with various combinations of plasmids or siRNA. At $24 \mathrm{~h}-36 \mathrm{~h}$ after the transfection, the cell lysates were prepared in RIPA lysis buffer plus a protease inhibitor 'cocktail' (Roche). MT2, MT4, C8166 and Jurkat cells were simulated by NaHS $(100 \mu \mathrm{mol} / \mathrm{L})$ for 2 days or 3 days. The cell lysates were incubated with indicated antibodies and analyzed by immunoblot.

\section{Real-time PCR}

Total RNA was extracted from the cultured cells with TRIzol reagent (Invitrogen) as described by the manufacturer. All gene transcripts were quantified by real-time PCR with the ABI 7500 Fast real-time PCR system (Applied Biosystems). The results was analyzed using the $2^{-\Delta \Delta C t}$ method. The primers used for real-time PCR were as follows:

Tax,

Forward, 5'-CGGATACCCAGTCTACGTGT-3', Reverse, 5'-GAGCCGATAACGCGTCCATCG-3';

GAPDH,

Forward, 5'-GCCAAAAGGGTCATCATCTC- 3', Reverse, 5'-GTAGAGGCAGGGATGATGTTC-3'.

\section{Transmission electron microscope}

After indicated stimulation, MT2 cells were fixed with the TEM fixative. And then let the precipitation re-suspended in the fixative and fixed at $4{ }^{\circ} \mathrm{C}$ for preservation and transportation. The operation was performed according to the instructions. The cuprum grids are observed under TEM (HITACHI: HT7800/HT7700) and take images.

\section{RNA interference}

The siRNA, CSE-silencer, was obtained from Genepharma. The sequences used were as follows: K1, forward, 5'-GCCGAGAGCUUGGGAGGAUTT-3', reverse, 5'AUCCUCCCAAGCUCUCGGCTT-3'; K2， forward,5'-GCAGCCACUGUAACUAUUATT-3', reverse, 5'-UAAUAGUUACAGUGGCUGCTT-3'. The negative control was purchased from Genepharma (catalog no. A06001).

\section{Co-culture}

Hela cells were cultured for 24 hours. MT2 cells were seeded and co-cultured with Hela cells at a ratio of 1:100 $\left(1 \times 10^{4} \mathrm{HTLV}-1\right.$ donor cells: $1 \times 10^{6}$ uninfected recipient cells ratio $)$ and allowed to incubate for an additional 24 hours prior to harvesting for downstream assays. 


\section{Statistical analysis}

The data are presented as the mean \pm standard error from at least three independent experiments. All p-values were calculated using two-tailed Student's t-tests (Graphpad) and were considered statistically no significant when $\mathrm{p}>0.05$.

\section{List of abbreviations}

HTLV-1: human T-cell leukemia virus type-1; $\mathrm{H}_{2} \mathrm{~S}$ : hydrogen sulfide; CSE: cystathionine $\gamma$-lyase; CBS: cystathionine $\beta$-synthase; MST: 3-mercaptopyruvate sulfur transferase; CO: carbon monoxide; NO: nitric oxide; ARE: Antioxidant Response Element; ATL: Adult T-cell leukemia; RA: rapamycin; 3-MA: 3-Methyladenine;

\section{Acknowledgements}

We are grateful to Dr. Liangwei Duan for the design of primers.

\section{Authors' Contributions}

H.L. and H.W. conceived the idea for the project. J.S., S.G., X.C., Z.Z., J.W., C.W., X.Z., L.Y. did the experiments. H.L. designed experiments, analyzed the results and wrote the paper.

Ethics approval and consent to participate

\section{Funding}

This work was supported by grants from Key scientific research projects in Universities of Henan Province (20A320011) and the National Natural Science Foundation of China (32000612).

\section{Availability of data and materials}

Not applicable.

\section{Declarations}

Ethics approval and consent to participate

Not applicable.

\section{Consent to publish}

Its publication has been approved by all co-authors.

\section{Competing interests}

The authors declare that they have no competing interests.

\section{Author details}

${ }^{1}$ Henan Key Laboratory of Immunology and Targeted Drugs, School of Laboratory Medicine, Xinxiang Medical University, Xinxiang, China.

${ }^{2}$ Henan Collaborative Innovation Center of Molecular Diagnosis and Laboratory Medicine, Xinxiang Medical University, Xinxiang, China.

${ }^{3}$ Seventh People's Hospital of Shanghai University of Traditional Chinese Medicine, Shanghai, China.

\section{References}

1. Wang, R., Two's company, three's a crowd: can H2S be the third endogenous gaseous transmitter? FASEB J 2002, 16 (13), 1792-8.

2. Li, L.; Moore, P. K., Putative biological roles of hydrogen sulfide in health and disease: a breath of not so fresh air? Trends Pharmacol Sci 2008, 29 (2), 84-90.

3. Szabo, C., Hydrogen sulphide and its therapeutic potential. Nat Rev Drug Discov 2007, 6 (11), 917 35. 
4. Fiorucci, S.; Distrutti, E.; Cirino, G.; Wallace, J. L., The emerging roles of hydrogen sulfide in the gastrointestinal tract and liver. Gastroenterology 2006, 131 (1), 259-71.

5. Zhao, W.; Zhang, J.; Lu, Y.; Wang, R., The vasorelaxant effect of $\mathrm{H}(2) \mathrm{S}$ as a novel endogenous gaseous K(ATP) channel opener. EMBO J 2001, 20 (21), 6008-16.

6. Abe, K.; Kimura, H., The possible role of hydrogen sulfide as an endogenous neuromodulator. $J$ Neurosci 1996, 16 (3), 1066-71.

7. Singh, S.; Padovani, D.; Leslie, R. A.; Chiku, T.; Banerjee, R., Relative contributions of cystathionine beta-synthase and gamma-cystathionase to $\mathrm{H} 2 \mathrm{~S}$ biogenesis via alternative trans-sulfuration reactions. J Biol Chem 2009, 284 (33), 22457-22466.

8. Chiku, T.; Padovani, D.; Zhu, W.; Singh, S.; Vitvitsky, V.; Banerjee, R., H2S biogenesis by human cystathionine gamma-lyase leads to the novel sulfur metabolites lanthionine and homolanthionine and is responsive to the grade of hyperhomocysteinemia. J Biol Chem 2009, 284 (17), 11601-12.

9. Beltowski, J., Synthesis, Metabolism, and Signaling Mechanisms of Hydrogen Sulfide: An Overview. Methods Mol Biol 2019, 2007, 1-8.

10. Bao, L.; Vlcek, C.; Paces, V.; Kraus, J. P., Identification and tissue distribution of human cystathionine beta-synthase mRNA isoforms. Arch Biochem Biophys 1998, 350 (1), 95-103.

11. Zuhra, K.; Augsburger, F.; Majtan, T.; Szabo, C., Cystathionine-beta-Synthase: Molecular Regulation and Pharmacological Inhibition. Biomolecules 2020, 10 (5).

12. Ishii, I.; Akahoshi, N.; Yu, X. N.; Kobayashi, Y.; Namekata, K.; Komaki, G.; Kimura, H., Murine cystathionine gamma-lyase: complete cDNA and genomic sequences, promoter activity, tissue distribution and developmental expression. Biochem J 2004, 381 (Pt 1), 113-23.

13. Jurkowska, H.; Kaczor-Kaminska, M.; Bronowicka-Adamska, P.; Wrobel, M., [Cystathionine gamma-lyase]. Postepy Hig Med Dosw (Online) 2014, 68, 1-9.

14. Shibuya, N.; Mikami, Y.; Kimura, Y.; Nagahara, N.; Kimura, H., Vascular endothelium expresses 3-mercaptopyruvate sulfurtransferase and produces hydrogen sulfide. J Biochem 2009, 146 (5), 623-6.

15. Shibuya, N.; Koike, S.; Tanaka, M.; Ishigami-Yuasa, M.; Kimura, Y.; Ogasawara, Y.; Fukui, K.; Nagahara, N.; Kimura, H., A novel pathway for the production of hydrogen sulfide from Dcysteine in mammalian cells. Nat Commun 2013, 4, 1366.

16. Marutani, E.; Morita, M.; Hirai, S.; Kai, S.; Grange, R. M. H.; Miyazaki, Y.; Nagashima, F.; Traeger, L.; Magliocca, A.; Ida, T.; Matsunaga, T.; Flicker, D. R.; Corman, B.; Mori, N.; Yamazaki, Y.; Batten, A.; Li, R.; Tanaka, T.; Ikeda, T.; Nakagawa, A.; Atochin, D. N.; Thara, H.; Olenchock, B. A.; Shen, X.; Nishida, M.; Hanaoka, K.; Kevil, C. G.; Xian, M.; Bloch, D. B.; Akaike, T.; Hindle, A. G.; Motohashi, H.; Ichinose, F., Sulfide catabolism ameliorates hypoxic brain injury. Nat Commun 2021, 12 (1), 3108.

17. Fuschillo, S.; Palomba, L.; Capparelli, R.; Motta, A.; Maniscalco, M., Nitric Oxide and Hydrogen Sulfide: A Nice Pair in the Respiratory System. Curr Med Chem 2020, 27 (42), 7136-7148.

18. Zhao, S.; Song, T.; Gu, Y.; Zhang, Y.; Cao, S.; Miao, Q.; Zhang, X.; Chen, H.; Gao, Y.; Zhang, L.; Han, Y.; Wang, H.; Pu, J.; Xie, L.; Ji, Y., Hydrogen Sulfide Alleviates Liver Injury Through the S-Sulfhydrated-Kelch-Like ECH-Associated Protein 1/Nuclear Erythroid 2-Related Factor 2/Low-Density Lipoprotein Receptor-Related Protein 1 Pathway. Hepatology 2021, 73 (1), 282302.

19. McCook, O.; Denoix, N.; Radermacher, P.; Waller, C.; Merz, T., H2S and Oxytocin Systems in Early Life Stress and Cardiovascular Disease. J Clin Med 2021, 10 (16). 
20. Szabo, C., Gasotransmitters in cancer: from pathophysiology to experimental therapy. Nat Rev Drug Discov 2016, 15 (3), 185-203.

21. Wang, R., Physiological implications of hydrogen sulfide: a whiff exploration that blossomed. Physiol Rev 2012, 92 (2), 791-896.

22. Hartle, M. D.; Pluth, M. D., A practical guide to working with H2S at the interface of chemistry and biology. Chem Soc Rev 2016, 45 (22), 6108-6117.

23. Paul, B. D.; Snyder, S. H., H2S: A Novel Gasotransmitter that Signals by Sulfhydration. Trends Biochem Sci 2015, 40 (11), 687-700.

24. Mustafa, A. K.; Gadalla, M. M.; Sen, N.; Kim, S.; Mu, W.; Gazi, S. K.; Barrow, R. K.; Yang, G.; Wang, R.; Snyder, S. H., H2S signals through protein S-sulfhydration. Sci Signal 2009, 2 (96), ra72.

25. Hourihan, J. M.; Kenna, J. G.; Hayes, J. D., The gasotransmitter hydrogen sulfide induces nrf2target genes by inactivating the keap1 ubiquitin ligase substrate adaptor through formation of a disulfide bond between cys-226 and cys-613. Antioxid Redox Signal 2013, 19 (5), 465-81.

26. Yang, G.; Zhao, K.; Ju, Y.; Mani, S.; Cao, Q.; Puukila, S.; Khaper, N.; Wu, L.; Wang, R., Hydrogen sulfide protects against cellular senescence via S-sulfhydration of Keap1 and activation of Nrf2. Antioxid Redox Signal 2013, 18 (15), 1906-19.

27. Matoba, K.; Noda, N. N., Structural catalog of core Atg proteins opens new era of autophagy research. J Biochem 2021, 169 (5), 517-525.

28. Nakatogawa, H., Mechanisms governing autophagosome biogenesis. Nat Rev Mol Cell Biol 2020, 21 (8), 439-458.

29. Mizushima, N.; Yoshimori, T.; Ohsumi, Y., The role of Atg proteins in autophagosome formation. Annu Rev Cell Dev Biol 2011, 27, 107-32.

30. Klionsky, D. J.; Schulman, B. A., Dynamic regulation of macroautophagy by distinctive ubiquitinlike proteins. Nat Struct Mol Biol 2014, 21 (4), 336-45.

31. Agrotis, A.; Pengo, N.; Burden, J. J.; Ketteler, R., Redundancy of human ATG4 protease isoforms in autophagy and LC3/GABARAP processing revealed in cells. Autophagy 2019, 15 (6), 976997.

32. Ketteler, R.; Tooze, S. A., ATG4: More Than a Protease? Trends Cell Biol 2021, 31 (7), 515-516.

33. Marino, G.; Uria, J. A.; Puente, X. S.; Quesada, V.; Bordallo, J.; Lopez-Otin, C., Human autophagins, a family of cysteine proteinases potentially implicated in cell degradation by autophagy. $J$ Biol Chem 2003, 278 (6), 3671-8.

34. Li, M.; Hou, Y.; Wang, J.; Chen, X.; Shao, Z. M.; Yin, X. M., Kinetics comparisons of mammalian Atg4 homologues indicate selective preferences toward diverse Atg8 substrates. J Biol Chem 2011, 286 (9), 7327-38.

35. Scherz-Shouval, R.; Sagiv, Y.; Shorer, H.; Elazar, Z., The COOH terminus of GATE-16, an intra-Golgi transport modulator, is cleaved by the human cysteine protease HsApg4A. J Biol Chem 2003, 278 (16), 14053-8.

36. Betin, V. M.; Lane, J. D., Caspase cleavage of Atg4D stimulates GABARAP-L1 processing and triggers mitochondrial targeting and apoptosis. J Cell Sci 2009, 122 (Pt 14), 2554-66.

37. Gallo, R. C., The discovery of the first human retrovirus: HTLV-1 and HTLV-2. Retrovirology 2005 , 2,17 .

38. Yoshida, M., Discovery of HTLV-1, the first human retrovirus, its unique regulatory mechanisms, and insights into pathogenesis. Oncogene 2005, 24 (39), 5931-7. 
39. Gessain, A.; Cassar, O., Epidemiological Aspects and World Distribution of HTLV-1 Infection. Front Microbiol 2012, 3, 388.

40. Goncalves, D. U.; Proietti, F. A.; Ribas, J. G.; Araujo, M. G.; Pinheiro, S. R.; Guedes, A. C.; Carneiro-Proietti, A. B., Epidemiology, treatment, and prevention of human T-cell leukemia virus type 1-associated diseases. Clin Microbiol Rev 2010, 23 (3), 577-89.

41. Tagaya, Y.; Matsuoka, M.; Gallo, R., 40 years of the human T-cell leukemia virus: past, present, and future. F1000Res 2019, 8 .

42. Futsch, N.; Mahieux, R.; Dutartre, H., HTLV-1, the Other Pathogenic Yet Neglected Human Retrovirus: From Transmission to Therapeutic Treatment. Viruses 2017, 10 (1).

43. Shimoyama, M., Diagnostic criteria and classification of clinical subtypes of adult T-cell leukaemialymphoma. A report from the Lymphoma Study Group (1984-87). Br J Haematol 1991, 79 (3), 428-37.

44. Tsukasaki, K.; Hermine, O.; Bazarbachi, A.; Ratner, L.; Ramos, J. C.; Harrington, W., Jr.; O'Mahony, D.; Janik, J. E.; Bittencourt, A. L.; Taylor, G. P.; Yamaguchi, K.; Utsunomiya, A.; Tobinai, K.; Watanabe, T., Definition, prognostic factors, treatment, and response criteria of adult T-cell leukemia-lymphoma: a proposal from an international consensus meeting. J Clin Oncol 2009, 27 (3), 453-9.

45. Ren, T.; Takahashi, Y.; Liu, X.; Loughran, T. P.; Sun, S. C.; Wang, H. G.; Cheng, H., HTLV-1 Tax deregulates autophagy by recruiting autophagic molecules into lipid raft microdomains. Oncogene 2015, 34 (3), 334-45.

46. Tang, S. W.; Chen, C. Y.; Klase, Z.; Zane, L.; Jeang, K. T., The cellular autophagy pathway modulates human T-cell leukemia virus type 1 replication. J Virol 2013, 87 (3), 1699-707.

47. Ducasa, N.; Grasso, D.; Benencio, P.; Papademetrio, D. L.; Biglione, M.; Kashanchi, F.; Berini, C.; Garcia, M. N., Autophagy in Human T-Cell Leukemia Virus Type 1 (HTLV-1) Induced Leukemia. Front Oncol 2021, 11, 641269.

48. Schaaf, M. B.; Keulers, T. G.; Vooijs, M. A.; Rouschop, K. M., LC3/GABARAP family proteins: autophagy-(un)related functions. FASEB J 2016, 30 (12), 3961-3978.

49. Till, A.; Subramani, S., A balancing act for autophagin. J Clin Invest 2010, 120 (7), 2273-6.

50. Sugawara, K.; Suzuki, N. N.; Fujioka, Y.; Mizushima, N.; Ohsumi, Y.; Inagaki, F., Structural basis for the specificity and catalysis of human Atg4B responsible for mammalian autophagy. J Biol Chem 2005, 280 (48), 40058-65.

51. Kumanomidou, T.; Mizushima, T.; Komatsu, M.; Suzuki, A.; Tanida, I.; Sou, Y. S.; Ueno, T.; Kominami, E.; Tanaka, K.; Yamane, T., The crystal structure of human Atg4b, a processing and de-conjugating enzyme for autophagosome-forming modifiers. J Mol Biol 2006, 355 (4), 612-8.

52. Robert, X.; Gouet, P., Deciphering key features in protein structures with the new ENDscript server. Nucleic Acids Res 2014, 42 (Web Server issue), W320-4.

53. Bazhanov, N.; Escaffre, O.; Freiberg, A. N.; Garofalo, R. P.; Casola, A., Broad-Range Antiviral Activity of Hydrogen Sulfide Against Highly Pathogenic RNA Viruses. Sci Rep 2017, 7, 41029. 54. Bazhanov, N.; Ivanciuc, T.; Wu, H.; Garofalo, M.; Kang, J.; Xian, M.; Casola, A., ThiolActivated Hydrogen Sulfide Donors Antiviral and Anti-Inflammatory Activity in Respiratory Syncytial Virus Infection. Viruses 2018, 10 (5).

55. Pal, V. K.; Bandyopadhyay, P.; Singh, A., Hydrogen sulfide in physiology and pathogenesis of bacteria and viruses. IUBMB Life 2018, 70 (5), 393-410. 
56. Citi, V.; Martelli, A.; Brancaleone, V.; Brogi, S.; Gojon, G.; Montanaro, R.; Morales, G.; Testai, L.; Calderone, V., Anti-inflammatory and antiviral roles of hydrogen sulfide: Rationale for considering H2 S donors in COVID-19 therapy. Br J Pharmacol 2020, 177 (21), 4931-4941.

57. Evgen'ev, M. B.; Frenkel, A., Possible application of H2S-producing compounds in therapy of coronavirus (COVID-19) infection and pneumonia. Cell Stress Chaperones 2020, 25 (5), 713-715.

58. Yang, G., H2S as a potential defense against COVID-19? Am J Physiol Cell Physiol 2020, 319 (2), C244-C249.

59. Qianqian ZHENG, L. D., Yang ZHANG, Jiaoyang LI, Shiyu ZHANG and Hui WANG, A dynamically evolving war between autophagy and pathogenic microorganisms. Journal of Zhejiang University SCIENCE B $\mathbf{2 0 2 1 .}$

60. Betin, V. M.; Singleton, B. K.; Parsons, S. F.; Anstee, D. J.; Lane, J. D., Autophagy facilitates organelle clearance during differentiation of human erythroblasts: evidence for a role for ATG4 paralogs during autophagosome maturation. Autophagy 2013, 9 (6), 881-93.

61. Huang, T.; Kim, C. K.; Alvarez, A. A.; Pangeni, R. P.; Wan, X.; Song, X.; Shi, T.; Yang, Y.; Sastry, N.; Horbinski, C. M.; Lu, S.; Stupp, R.; Kessler, J. A.; Nishikawa, R.; Nakano, I.; Sulman, E. P.; Lu, X.; James, C. D.; Yin, X. M.; Hu, B.; Cheng, S. Y., MST4 Phosphorylation of ATG4B Regulates Autophagic Activity, Tumorigenicity, and Radioresistance in Glioblastoma. Cancer Cell 2017, 32 (6), 840-855 e8.

62. Pengo, N.; Agrotis, A.; Prak, K.; Jones, J.; Ketteler, R., A reversible phospho-switch mediated by ULK1 regulates the activity of autophagy protease ATG4B. Nat Commun 2017, 8 (1), 294.

63. Ni, Z.; He, J.; Wu, Y.; Hu, C.; Dai, X.; Yan, X.; Li, B.; Li, X.; Xiong, H.; Li, Y.; Li, S.; Xu, L.; Li, Y.; Lian, J.; He, F., AKT-mediated phosphorylation of ATG4B impairs mitochondrial activity and enhances the Warburg effect in hepatocellular carcinoma cells. Autophagy 2018, 14 (4), 685-701.

64. Li, Y.; Zhang, Y.; Wang, L.; Wang, P.; Xue, Y.; Li, X.; Qiao, X.; Zhang, X.; Xu, T.; Liu, G.; Li, P.; Chen, C., Autophagy impairment mediated by S-nitrosation of ATG4B leads to neurotoxicity in response to hyperglycemia. Autophagy 2017, 13 (7), 1145-1160.

65. Zheng, X.; Yang, Z.; Gu, Q.; Xia, F.; Fu, Y.; Liu, P.; Yin, X. M.; Li, M., The protease activity of human ATG4B is regulated by reversible oxidative modification. Autophagy 2020, 16 (10), 1838-1850. 